\title{
Local Radiation Therapy Inhibits Tumor Growth through the Generation of Tumor-Specific CTL: Its Potentiation by Combination with Th1 Cell Therapy
}

\author{
Tsuguhide Takeshima ${ }^{1}$, Kenji Chamoto $^{2}$, Daiko Wakita $^{3}$, Takayuki Ohkuri ${ }^{2}$, Yuji Togashi ${ }^{4}$, \\ Hiroki Shirato ${ }^{1}$, Hidemitsu Kitamura ${ }^{2}$, and Takashi Nishimura ${ }^{2,3}$
}

\section{Abstract}

Radiation therapy is one of the primary treatment modalities for cancer along with chemotherapy and surgical therapy. The main mechanism of the tumor reduction after irradiation has been considered to be damage to the tumor DNA. However, we found that tumor-specific CTL, which were induced in the draining lymph nodes (DLN) and tumor tissue of tumor-bearing mice, play a crucial role in the inhibition of tumor growth by radiation. Indeed, the therapeutic effect of irradiation was almost completely abolished in tumor-bearing mice by depleting $\mathrm{CD}^{+} \mathrm{T}$ cells through anti-CD8 monoclonal antibody administration. In mice whose DLN were surgically ablated or genetically defective (Aly/Aly mice), the generation of tetramer ${ }^{+}$tumor-specific CTL at the tumor site was greatly reduced in parallel with the attenuation of the radiation-induced therapeutic effect against the tumor. This indicates that DLN are essential for the activation and accumulation of radiationinduced CTL, which are essential for inhibition of the tumor. A combined therapy of local radiation with Th1 cell therapy augmented the generation of tumor-specific CTL at the tumor site and induced a complete regression of the tumor, although radiation therapy alone did not exhibit such a pronounced therapeutic effect. Thus, we conclude that the combination treatment of local radiation therapy and Th1 cell therapy is a rational strategy to augment antitumor activity mediated by tumor-specific CTL. Cancer Res; 70(7); 2697-706. @2010 AACR.

\section{Introduction}

Radiation therapy is one of the major modalities for the treatment of human cancer and has been established as an excellent local treatment for malignant tumors (1). However, radiotherapy does not always achieve the same level of efficacy against tumors as surgery or chemotherapy. It has been considered that the main mechanism of the tumor reduction after irradiation is direct damage to the tumor DNA by ionizing irradiation. Recently, however, some studies have reported that tumor local irradiation elicits immunomodulatory effects and induces tumor-specific immune responses (2-6). Lugade and colleagues (2) showed that local radiation increased both the generation of tumor peptide-reactive IFN- $\gamma$-producing antitumor immune cells and their traffick-

Authors' Affiliations: 1 Department of Radiology, Hokkaido University Graduate School of Medicine; 2Division of Immunoregulation and ${ }^{3}$ ROYCE' Health Bioscience, Section of Disease Control, Institute for Genetic Medicine, Hokkaido University; and "Bioimmulance Co. Ltd., Sapporo, Japan

Note: Supplementary data for this article are available at Cancer Research Online (http://cancerres.aacrjournals.org/).

T. Takeshima and K. Chamoto contributed equally to this work.

Corresponding Author: Takashi Nishimura, Division of Immunoregulation, Section of Disease Control, Institute for Genetic Medicine, Hokkaido University, Sapporo 060-0815, Japan. Phone/Fax: 81-011-706-7546; E-mail: tak24@igm.hokudai.ac.jp.

doi: 10.1158/0008-5472.CAN-09-2982

(C)2010 American Association for Cancer Research. ing to the tumor-draining lymph nodes (DLN) and tumor tissues. Apetoh and colleagues $(3,5)$ clearly showed the essential role of $\mathrm{T}$ cells in radiation therapy by comparing the tumor growth between T-cell-deficient nude mice and immunocompetent wild-type mice. They also showed that TLR4 on host dendritic cells was crucial to induce antitumor T cells. Recently, Lee and colleagues (6) reported the essential role of $\mathrm{CD} 8^{+} \mathrm{T}$ cells in radiation-induced tumor growth inhibition using anti-CD8 monoclonal antibody (mAb) treatment. Moreover, they showed that radiation induced the activation of T-cell receptor (TCR) tetramer-positive dendritic cells, which could induce a rapid proliferation of antigen-specific $2 \mathrm{C}$-derived $\mathrm{T}$ cells in tumor DLN. Therefore, $\mathrm{CD}^{+} \mathrm{T}$ cells, IFN- $\gamma$-producing cells, the interaction of tumor antigen and TLR4 on dendritic cells, and T-cell proliferation in tumor DLN have all been shown to play important roles in the radiation treatment of tumors.

In this article, we initially showed a close relationship between the accumulation of radiation-induced tumor-specific $\mathrm{CD}^{+} \mathrm{CTL}$ at local tumor sites and radiation-induced tumor growth inhibition. We also showed that tumor DLN are indispensable organs for inducing radiation-induced tumorspecific CTL, which are essential for inhibiting tumor growth by radiation. Namely, radiation-induced tumor growth inhibition was canceled in surgically lymph node (LN)-ablated mice or LN-deficient Aly/Aly mice.

This work was greatly expanded by our finding that the combination of radiotherapy and Th1 cell therapy is a novel strategy to augment the systemical antitumor activity mediated 
by tumor-specific CTL, and that it might also be effective for the treatment of distant metastases. Previously, we showed that the introduction of tumor-specific Thl-dominant immunity is crucial for inducing tumor-specific CTL, which are essential for a complete cure of tumor-bearing mice (7-9). This concept has been supported by the accumulating data of many investigators based on both animal and clinical studies (10-19). Thus, we believe that the combination treatment of local radiation therapy and Thl cell therapy will become a rational strategy to eradicate tumors through the augmented induction of $\mathrm{CD} 8^{+}$tumorspecific CTL at the tumor DLN and tumor tissue.

\section{Materials and Methods}

Mice. Female C57BL/6 mice were obtained from Charles River Japan. Female alymphoplasia-mutant mice (Aly/Aly mice, C57BL/6 background), which are characterized by the systemic absence of lymph nodes and Peyer's patches (20), were purchased from CLEA Japan. Female $\mathrm{Pfp}^{-/-}$(perforin knockout) mice were obtained from Taconic (21). All mice were female and were used at 6 to $8 \mathrm{wk}$ of age and maintained in specific pathogen-free conditions.

Reagents. Interleukin 2 (IL-2) was supplied by T. Sawada (Shionogi Pharmaceutical Institute Co. Ltd., Osaka, Japan). IL-12 and IFN- $\gamma$ were purchased from PeproTech EC Ltd. FITC-CD8 mAb was purchased from Beckman Coulter, and phycoerythrin (PE)-Cy7-CD8 mAb and FITC-IFN- $\gamma$ $\mathrm{mAb}$ were purchased from $\mathrm{BD}$ Bioscience. Ovalbumin protein (OVA) was purchased from Sigma-Aldrich Japan. H$2 \mathrm{~K}^{\mathrm{b}}$ OVA tetramer-SIINFEKL-PE (OVA tetramer) and $\mathrm{H}-2 \mathrm{~K}^{\mathrm{b}}$ TRP-2 tetramer-SVYDFFVWL-PE (TRP-2 tetramer) were purchased from MBL.

Cell culture. Tumor cell lines, EL4 lymphoma, EG7 (EL4 transduced to express OVA), Lewis lung carcinoma (LLC), LLC-OVA (LLC transduced to express OVA), B16 melanoma, and lymphocytes from the DLN were cultured in RPMI 1640 supplemented with $10 \%$ FCS, $2 \mathrm{mmol} / \mathrm{L} \mathrm{L}$-glutamine, $0.05 \mathrm{mmol} / \mathrm{L} 2$-mercaptoethanol, HEPES, penicillin, and streptomycin at $37^{\circ} \mathrm{C}$ in a humidified atmosphere containing $5 \% \mathrm{CO}_{2}$. TRP-2-tetramer ${ }^{+} \mathrm{CD}^{+}$cells in DLN were induced as follows. DLN were removed $7 \mathrm{~d}$ after irradiation and DLN cells were restimulated with mitomycin $\mathrm{C}$ (MMC)-treated B16 cells (stimulator/responder ratio, 1:10) in the presence of IL-2 (100 units/mL), IL-12 (20 units/mL), and IFN- $\gamma(1 \mathrm{ng} / \mathrm{mL})$ for $12 \mathrm{~d}$.

Local irradiation. EG7, LLC-OVA, and B16 melanoma cells $\left(2 \times 10^{6}\right.$ per mouse) were intradermally (i.d.) inoculated into the right hind limb. When the tumor mass became large (6-8 $\mathrm{mm}$ in diameter) at $7 \mathrm{~d}$ of tumor inoculation, the tumorbearing mice were anesthetized $(50 \mathrm{mg} / \mathrm{kg}$ Nembutal) and fixed in a plastic syringe with a hole. The right leg with the target tumor was then pulled out of the syringe through the hole, and the mouse was placed under a 4-mm-thick lead shield excluding the right leg tumor area. Then, the right leg of the tumor-bearing mouse was irradiated once with 2 Gy (EG7) or 15 Gy (LLC-OVA and B16) while shielding the DLN with the lead shield ( $150 \mathrm{kV}, 2 \mathrm{~Gy} / \mathrm{min}$; Gemini ver- tical X-ray beam with an aluminum 2-mm filter, Hitachi Medico). Different tumors were given different doses according to their radiosensitivity (Supplementary Fig. S1). The antitumor activity was determined by measuring the tumor size in perpendicular diameters. The tumor volume was calculated by the following formula: tumor volume $=0.4 \times$ length $(\mathrm{mm}) \times[\text { width }(\mathrm{mm})]^{2}$.

Antibody staining and flow cytometry. For the analysis of tumor-specific CTL frequencies, lymphocytes from the DLN and tumor tissue were stained with FITC-conjugated antiCD8 mAb and PE-conjugated OVA tetramer or PE-conjugated TRP-2 tetramer. Data were acquired on a FACSCalibur (Becton Dickinson) and analyzed using CellQuest software (Becton Dickinson). The method used to detect cytoplasmic IFN- $\gamma$ was described in our previous article (22). Here, staining was done with PE-Cy7-CD8 and FITC-conjugated anti-IFN- $\gamma$ mAbs.

Cytotoxicity assay. The cytotoxicity mediated by tumorspecific CTL was measured by a $4-\mathrm{h}{ }^{51} \mathrm{Cr}$-release assay as previously described (23). To confirm the antigen specificity of the $\mathrm{H}-2 \mathrm{~K}^{\mathrm{b}}$-restricted CTL, ${ }^{51} \mathrm{Cr}$-labeled target cells were incubated with $\mathrm{CD}^{+}$CTL pretreated with OVA tetramer, which can block the recognition of $\mathrm{H}-2 \mathrm{~K}^{\mathrm{b}}$-restricted target peptide $\left(\mathrm{OVA}_{257-264}\right)$ by CTL; this method was termed tetramerblocking assay (24). The percent cytotoxicity was calculated as previously described (23).

In vivo CD8+ cells and IFN- $\gamma$ depletion. To deplete $\mathrm{CD}^{+}$ $\mathrm{T}$ cells in mice, tumor-bearing mice were injected i.v. with $200 \mu \mathrm{g}$ of purified anti-CD8 mAb (53-6.7) after irradiation at intervals of $5 \mathrm{~d}$. To examine the role of IFN- $\gamma$ in mice, tumor-bearing mice were injected i.p. with $200 \mu \mathrm{g}$ of neutralizing anti-IFN- $\gamma \mathrm{mAb}$ (R4-6A2) $12 \mathrm{~h}$ before the irradiation and subsequently at 3-d intervals.

Surgical excision of tumor DLN. Mice were anesthetized. A small incision was made in the skin at the inguinal region. The inguinal lymph nodes were removed with tweezers and the incision was closed with two clips. At the end of each experiment, the mice were killed under Nembutal anesthesia.

Generation of OVA-specific ThI cells from OT-II TCR transgenic mice. The method for generation of OVA-specific Thl cells was previously described (25). $\mathrm{CD}^{+} \mathrm{CD}^{+} 5 \mathrm{RB}^{+}$naive $\mathrm{T}$ cells were isolated from spleen cells of OT-II TCR transgenic mice and were stimulated with $\mathrm{OVA}_{323-339}$ peptide in the presence of MMC-treated spleen cells, IL-2, IL-12, IFN- $\gamma$, and anti-IL-4 mAb for Th1 development. At $48 \mathrm{~h}$, cells were restimulated with $\mathrm{OVA}_{323-339}$ under the same conditions and used at 9 to $12 \mathrm{~d}$ of culture.

Tumor radiotherapy combined with an immunotherapy model. EG7-bearing mice were irradiated as described above. Just after irradiation, a mixture of OVA protein $(200 \mu \mathrm{g} /$ mouse) and OVA-specific Th1 cells $\left(5 \times 10^{6}\right.$ per mouse $)$ was i.d. injected in proximity to the tumor DLN. Injection of OVA and OVA-specific Thl cells was done three times every $2 \mathrm{~d}$. Antitumor activity was determined by measuring the tumor size in perpendicular diameters. Tumor volume was calculated in the manner described above. Tumorbearing mice that survived for $>100 \mathrm{~d}$ after therapy were considered completely cured. For contralateral experiments 
(a metastatic model), EG7 cells were i.d. injected into the right leg $7 \mathrm{~d}$ before starting the combination therapy and into the left leg $2 \mathrm{~d}$ before the therapy. When the tumor mass of the right leg became large, the therapy was done only on the right leg as described above, and then the volumes of both tumors were measured.

\section{Results}

Generation of tumor-specific CTL by local tumor irradiation. We examined whether tumor-specific CTL could be induced by local tumor irradiation. EG7- or LLC-OVA-bearing mice were irradiated with a single dose of 2 or $15 \mathrm{~Gy}$, respectively. The difference in the optimal radiation doses between EG7 and LLC-OVA was due to their different radiosensitivities (Supplementary Fig. S1). Then, we analyzed the frequency of tumor-specific CTL generation in DLN and tumor tissue 5 days after irradiation. Lymphocytes were isolated from tu- mor DLN and tumor mass to examine the generation of model tumor antigen (OVA)-specific CTL by staining with $\mathrm{OVA} / \mathrm{H}-2 \mathrm{~K}^{\mathrm{b}}$ tetramer. The frequency of OVA-tetramer ${ }^{+} \mathrm{CD} 8^{+}$ CTL in EG7-bearing mice treated with radiation therapy was markedly elevated in tumor DLN (from 0.6-1.7\%) and tumor tissue (from 4.3-14.2\%) compared with sham-irradiated mice or EL4-irradiated mice $\left[0.4 \%\right.$ in DLN and $0.5 \%$ in $\mathrm{CD}^{+}$ tumor-infiltrating T lymphocytes (TIL); Fig. 1A]. Similarly, the frequency of OVA-tetramer ${ }^{+} \mathrm{CD}^{+} \mathrm{CTL}$ in LLC-OVA mice was also elevated in tumor DLN (from $0.7 \%$ to $1.4 \%$ ) and tumor tissue (from $4.8 \%$ to $15.3 \%$ ) compared with the levels in sham-irradiated mice or LLC-irradiated mice $(0.3 \%$ in DLN and TIL; Fig. 1B). The total lymphocyte population in DLN isolated from EG7-bearing or LLC-OVA mice exhibited a specific cytotoxicity against transplanted EG7 or LLC-OVA tumor cells but not against their parental EL4 or LLC tumor cells, respectively (Fig. 1C and D). Moreover, the cytotoxicity of radiation-induced CTL in DLN of tumor-bearing mice was
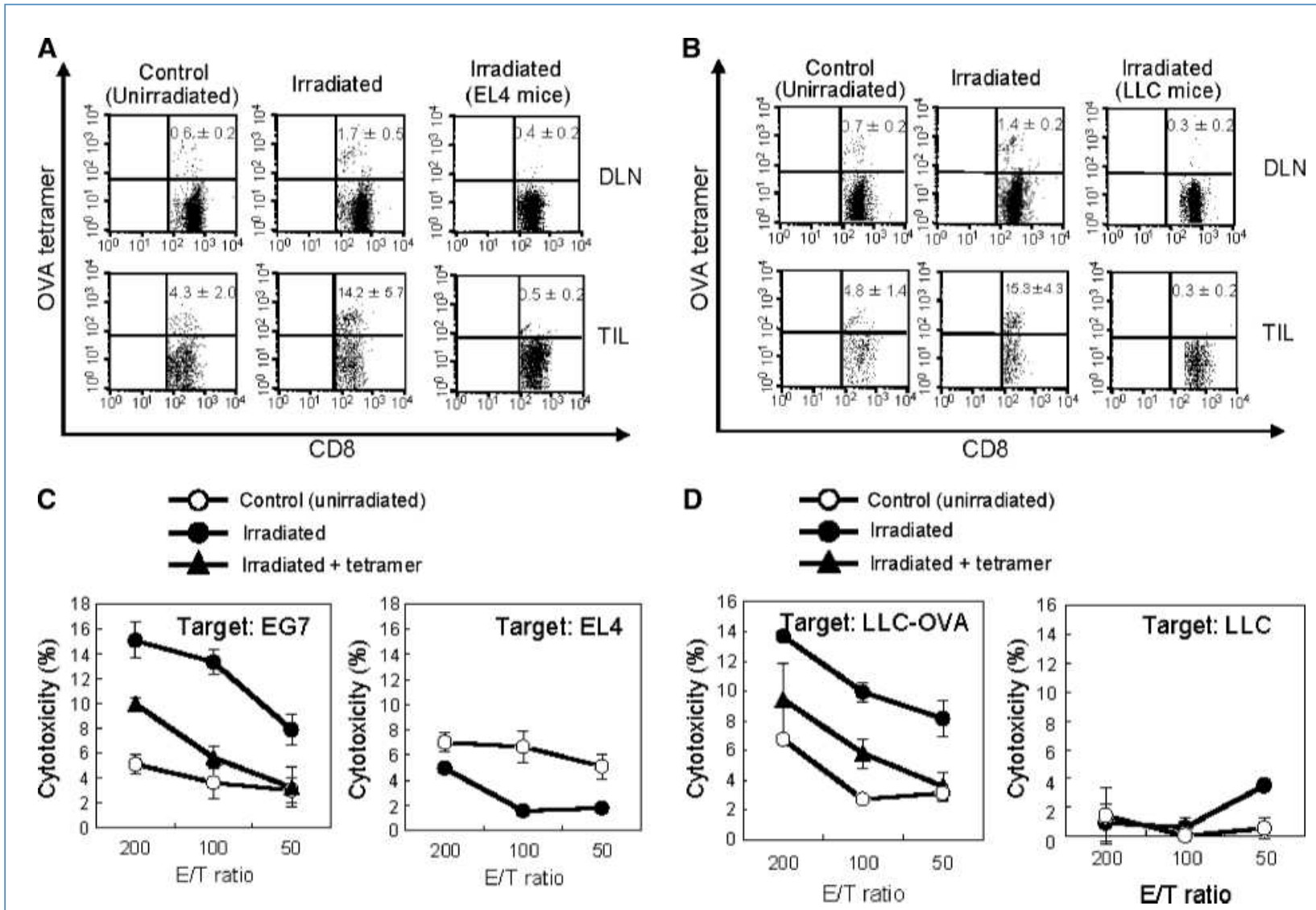

Figure 1. Induction of tumor model antigen-specific CTL by local tumor irradiation. Both EG7- and EL4-bearing mice were irradiated with 2 Gy or both LLC-OVA- and LLC-bearing mice were irradiated with 15 Gy $7 \mathrm{~d}$ after tumor inoculation. A and B, generation of tumor-specific CTL in EG7-bearing (A) and LLC-OVA-bearing mice (B). DLN and TIL were harvested $5 \mathrm{~d}$ after irradiation and the percentage of tumor-specific CTL was investigated by staining with OVA tetramer. The numbers in the top right quadrant represent the mean $\pm \mathrm{SD}$ percentage of tetramer ${ }^{+} \mathrm{CD}^{+} \mathrm{T}$ cells among the total $\mathrm{CD} 8^{+} \mathrm{T}$ cells of three mice in each experimental group. $C$ and D, cytotoxicity of total DLN against parental or irrelevant tumor cell. The cytotoxicity of the total DLN cells prepared from EG7- (C) and LLC-OVA-bearing mice (D) was examined against EG7 or EL4 cells (C) and LLC-OVA or LLC cells (D), respectively. To determine the specificity of cytolysis, a tetramer-blocking assay was done ( $\mathbf{\Delta})$. Points, mean of triplicate samples; bars, SD. Similar results were obtained from three different experiments. E/T, effector/target. 


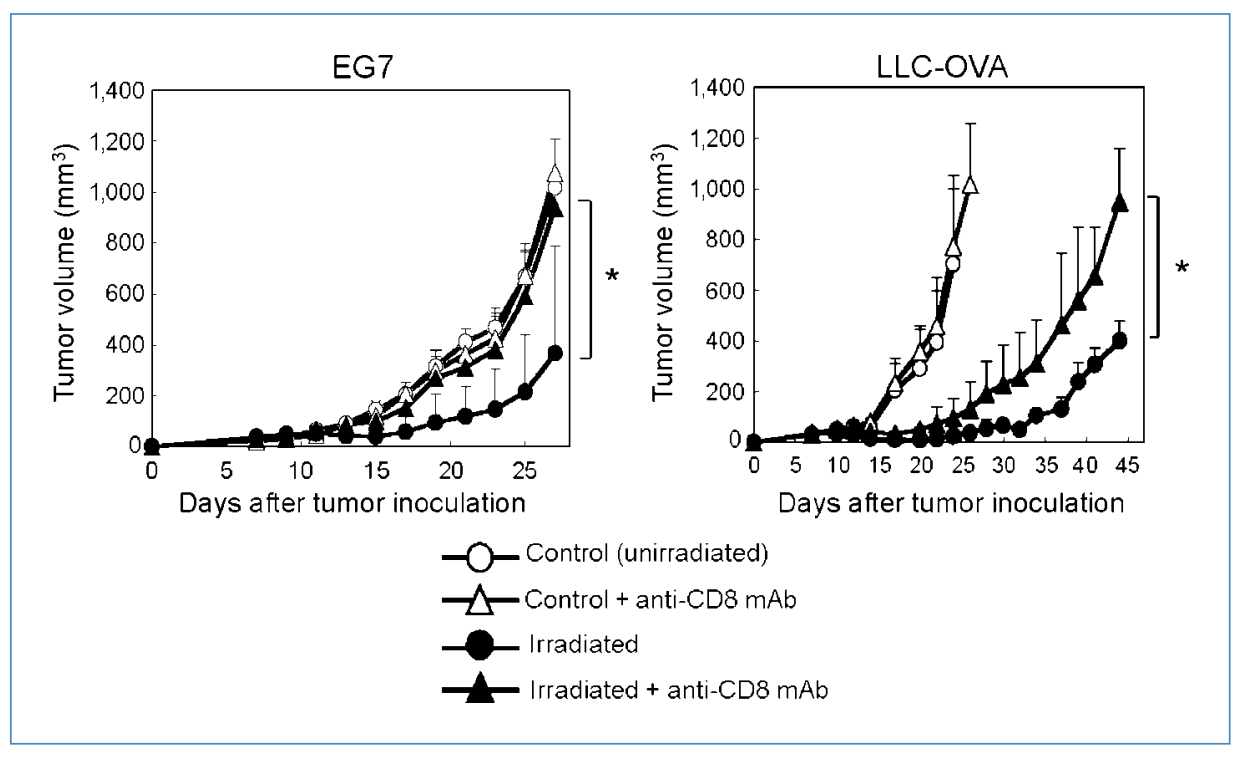

Figure 2. $\mathrm{CD}^{+} \mathrm{T}$ cells are essential for radiation therapy. EG7- or LLC-OVA-bearing mice were irradiated ( $\bullet$ and $\boldsymbol{\Lambda}$ ) or unirradiated $(O$ and $\Delta)$ and treated with i.v. injection of saline ( $O$ and $\bullet$ ) or anti-CD8 $\mathrm{mAb}(\boldsymbol{\Delta}$ and $\Delta$ ). The tumor volume was calculated as described in Materials and Methods. Points, mean of five mice in each experimental group; bars, SD. Similar results were obtained from three separate experiments. *, $P<0.05$, two-tailed Student's $t$ test. completely inhibited by adding OVA $/ \mathrm{H}-2 \mathrm{~K}^{\mathrm{b}}$ tetramer into the culture (tetramer-blocking assay; ref. 24). We also showed that IFN- $\gamma$ production of $\mathrm{CD}^{+} \mathrm{T}$ cells in TIL was significantly enhanced by irradiation (Supplementary Fig. S2).

Taken together, these data showed that local radiation therapy targeting the tumor mass effectively induced a functionally significant level of tumor-specific $\mathrm{CD} 8^{+} \mathrm{CTL}$ at the local tumor site.

Essential role of radiation-induced CD8+ tumor-specific CTL in the inhibition of tumor growth by local radiation therapy. To determine the critical role of tumor-specific CTL induced by localized tumor irradiation generally, we examined the effect of anti-CD8 mAb administration on the inhibition of tumor growth by radiation therapy using three kinds of tumors. As shown in Fig. 2A, treatment of EG7-bearing mice with local radiation therapy caused a significant inhibition of tumor growth compared with that of nontreated mice. However, administration of anti-CD8 mAb into tumorbearing mice to deplete $\mathrm{CD} 8^{+} \mathrm{T}$ cells resulted in a cancellation of radiation-induced tumor growth inhibition. We also observed similar results in LLC-OVA-bearing mice (Fig. 2B).

To generalize this phenomenon to natural tumor antigen systems, we next examined whether CTL that were specific to the natural tumor antigen TRP-2 could be induced by radiotherapy using B16-bearing mice. The frequency of tumor-specific CTL in tumor-bearing mice was analyzed 6 days after irradiation. Consistent with the results in EG7- or LLC-OVA-bearing mice (Fig. 1A), the frequency of TRP-2-specific CTL, which were stained with TRP- $2 / \mathrm{H}-2 \mathrm{~K}^{\mathrm{b}}$ tetramer, increased in DLN and tumor tissue by treatment with radiation therapy (Fig. 3A). Radiation-induced CTL exhibited TRP-2-specific cytotoxicity against B16 cells but not against TRP-2-negative LLC (Fig. 3B). The therapeutic effects of radiation therapy against B16 cells were greatly reduced by the administration of anti-CD8 $\mathrm{mAb}$ (Fig. 3C). Thus, we concluded that the generation of tumorspecific $\mathrm{CD}^{+} \mathrm{CTL}$ was essential for the inhibition of tumor growth by local radiation therapy not only in the EG7 or
LLC-OVA (tumor model antigen) system but also in the B16 (natural tumor antigen) system.

In addition, we examined the role of perforin in radiation therapy using $\mathrm{Pfp}^{-/-}$mice. As shown in Supplementary Fig. S3, treatment of EG7-bearing $\mathrm{Pfp}^{-/-}$mice with irradiation caused partial, but not total, inhibition of tumor growth compared with irradiated wild-type mice. Perforin is therefore an important agent that could help contribute to the reduction of tumor progression in radiation therapy, but other lytic molecules may be also involved in the therapy.

Critical role of tumor $\mathrm{DLN}$ in the generation of $\mathrm{CDS}+$ tumor-specific CTL by local radiation therapy. It has been reported that tumor DLN are indispensable for the generation of tumor-specific CD8 ${ }^{+} \mathrm{CTL}$ in the tumor mass during immunotherapy (8). To examine whether DLN are essential for the generation of radiation-induced CTL, the frequency of tumor-specific CTL in tumor tissue after radiation therapy was determined using surgically DLN-ablated mice or genetically LN-deficient Aly/Aly mice. As shown in Fig. 4A, in contrast to sham-operated tumor-bearing mice, the therapeutic effect of local tumor irradiation markedly decreased in tumor-bearing mice whose DLN were surgically aborted. The therapeutic effect of radiation therapy also decreased in Aly/Aly mice compared with that in wild-type mice (Fig. 4B). Consistent with this finding, local radiation induced no significant increase of tetramer ${ }^{+}$CTL in DLN-ablated mice or LN-deficient Aly/Aly mice, although a marked increase of tumor-specific CTL was observed in nontreated or shamoperated tumor-bearing mice after radiation therapy (Fig. 4C and D). These results suggest that tumor DLN are indispensable lymphoid organs for the induction of tumor-specific CTL critical for tumor radiotherapy.

Combination treatment of local radiation therapy and Th1 cell therapy is a rational strategy to augment the generation of systemic tumor-specific CTL in tumor-bearing mice. We next tried to develop a novel combination therapy with local radiation and Thl cell therapy. After irradiation 


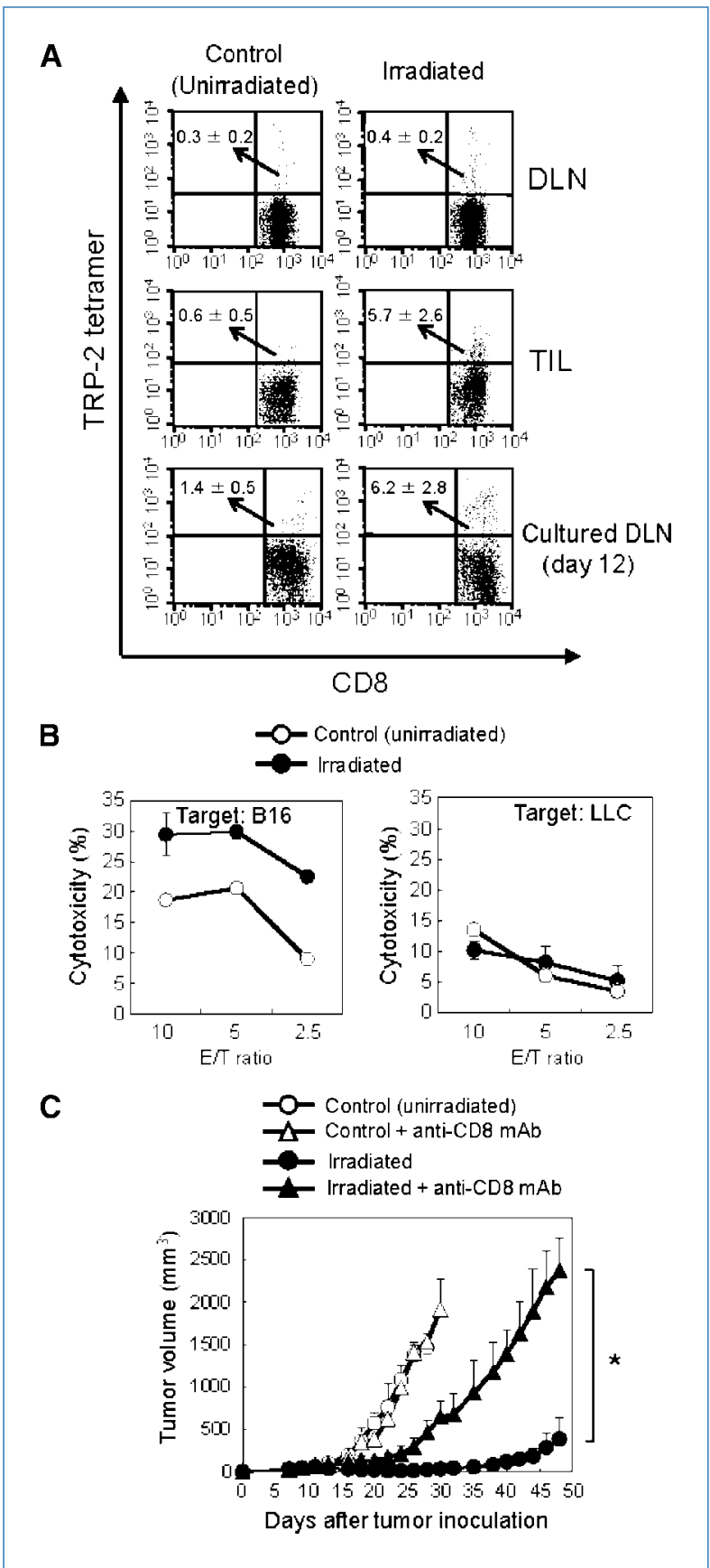

Figure 3. Induction of TRP-2-specific CTL by local tumor irradiation. A, TRP-2-specific CTL frequency in the DLN and TIL was detected by flow cytometry $7 \mathrm{~d}$ after local tumor irradiation of 15 Gy to B16-bearing mice. The method for preparation of cultured DLN was as described in Materials and Methods. The numbers in the top right quadrant represent the mean $\pm \mathrm{SD}$ percentage of tetramer ${ }^{+} \mathrm{CD}^{+} \mathrm{T}$ cells of the total CD8 ${ }^{+}$ $T$ cells of four mice in each experimental group. $B$, the cytotoxicity of the total DLN cells was examined in a $4-h{ }^{51} \mathrm{Cr}$-release assay. Points, mean of triplicate samples; bars, SD. C, B16-bearing mice were irradiated $(\bullet$ and $\Delta$ ) or unirradiated ( $O$ and $\Delta) 7 \mathrm{~d}$ after tumor inoculation and treated with i.v. injection of saline ( $\mathrm{O}$ and $\bullet$ ) or anti-CD8 $\mathrm{mAb}$ ( $\Delta$ and $\mathbf{\Delta}$ ). Points, mean of five mice in each experimental group; bars, SD. Similar results were obtained from three different experiments $(A-C)$. *, $P<0.05$, two-tailed Student's $t$ test. to EG7-bearing mice, Th1 therapy was done as described in Materials and Methods. Lymphocytes were prepared from DLN or tumor tissue to examine the generation of OVAspecific CTL on day 10. The frequency of tetramer ${ }^{+} \mathrm{CD}^{+}$ CTL in mice treated with the combination treatment of local radiation and Th1 cell therapy was markedly elevated in tumor tissue $(5.6 \%)$ compared with that in mice treated with other therapies (Fig. 5A). The cytotoxic activities of cells recovered from DLN were also measured using ${ }^{51} \mathrm{Cr}$-labeled OVA-positive EG7 cells or their parental OVA-negative EL4 tumor cells. The DLN cells from the mice treated with radiotherapy combined with $\mathrm{Th} 1$ cell therapy showed higher cytotoxicity against EG7 cells than did the DLN cells of other groups (Fig. 5B). It was also shown that the cytotoxicity of CTL from the combined therapy was strongly blocked by the OVA/H-2 $\mathrm{K}^{\mathrm{b}}$ tetramer (data not shown). No significant enhancement of cytotoxicity against OVA-negative parental EL4 tumor cells was detected in any experimental groups (Fig. 5B). We also showed that the frequency of IFN$\gamma$-producing $\mathrm{CD}^{+} \mathrm{T}$ cells increased in mice treated with irradiation and Thl cells plus OVA (22.4\%; Fig. 5C) compared with other groups.

Next, we examined the therapeutic efficacy of the combined treatment of radiotherapy and Th1 cell therapy. As shown in Fig. 6A, the combination therapy caused a profound growth inhibition of the established tumor mass and the tumor was completely eradicated in more than $80 \%$ of tumor-bearing mice (Fig. 6A and B). No significant therapeutic effect was observed in the other therapeutic protocols. To investigate whether the combination therapy could induce tumor-specific CTL systemically, we used the metastatic model described in Materials and Methods. In short, EG7 was inoculated into both legs and the combination therapy was done only on the right leg. As shown in Fig. $6 \mathrm{C}$, the tumor growth of the left leg was greatly inhibited along with that of the right leg in the group receiving the combination therapy (four of five mice were completely cured). Finally, we examined whether the combination therapy could induce immunologic memory for CTL. The mice in which EG7 tumors were completely eradicated by combination therapy were rechallenged with syngeneic EG7 or irrelevant LLC, and the growth of the tumor was monitored. As shown in Fig. 6D, EG7, but not LLC, was rejected, indicating that the mice acquired immunologic memory against syngeneic tumor cells.

Finally, we determined the role of IFN- $\gamma$ in the induction of OVA-tetramer ${ }^{+} \mathrm{CD} 8^{+} \mathrm{T}$ cells and on tumor growth after irradiation using anti-IFN- $\gamma$-neutralizing $\mathrm{mAb}$ or control rat anti-IgG antibody. As shown in Supplementary Figs. S4 and S5, anti-IFN- $\gamma$ mAb administration caused no significant inhibition of radiation-induced generation of OVA-tetramer ${ }^{+}$ $\mathrm{CD}^{+} \mathrm{T}$ cells in DLN and TIL or of the growth of EG7 tumor cells.

These results suggest that the combination treatment of local radiation therapy and Thl cell therapy is a rational strategy to augment the generation of tumor-specific CTL (Tc1) systemically essential for the rejection of tumor and for the prevention of metastasis and recurrence. 

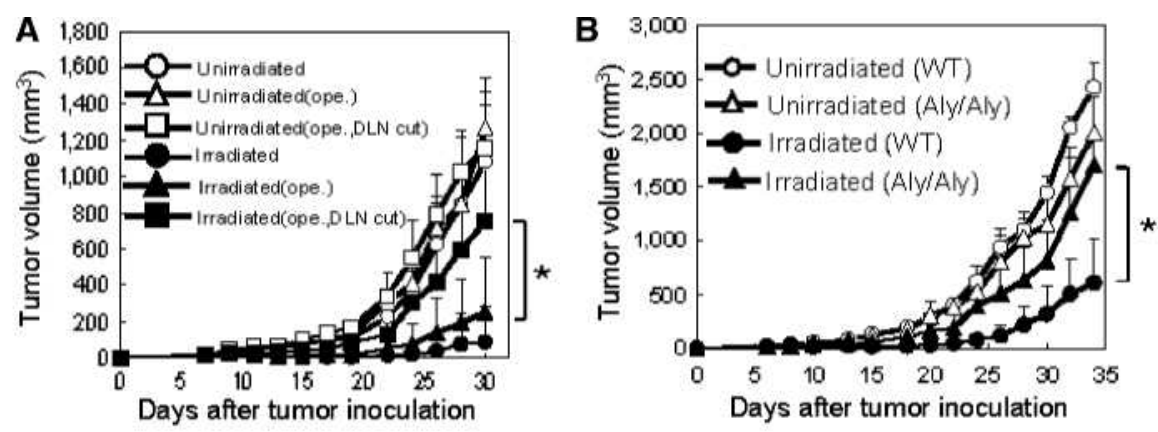

C

WT
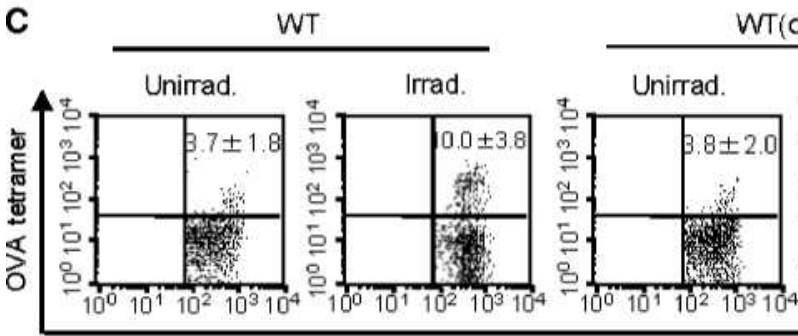

WT(ope.)

Days after tumor inoculation
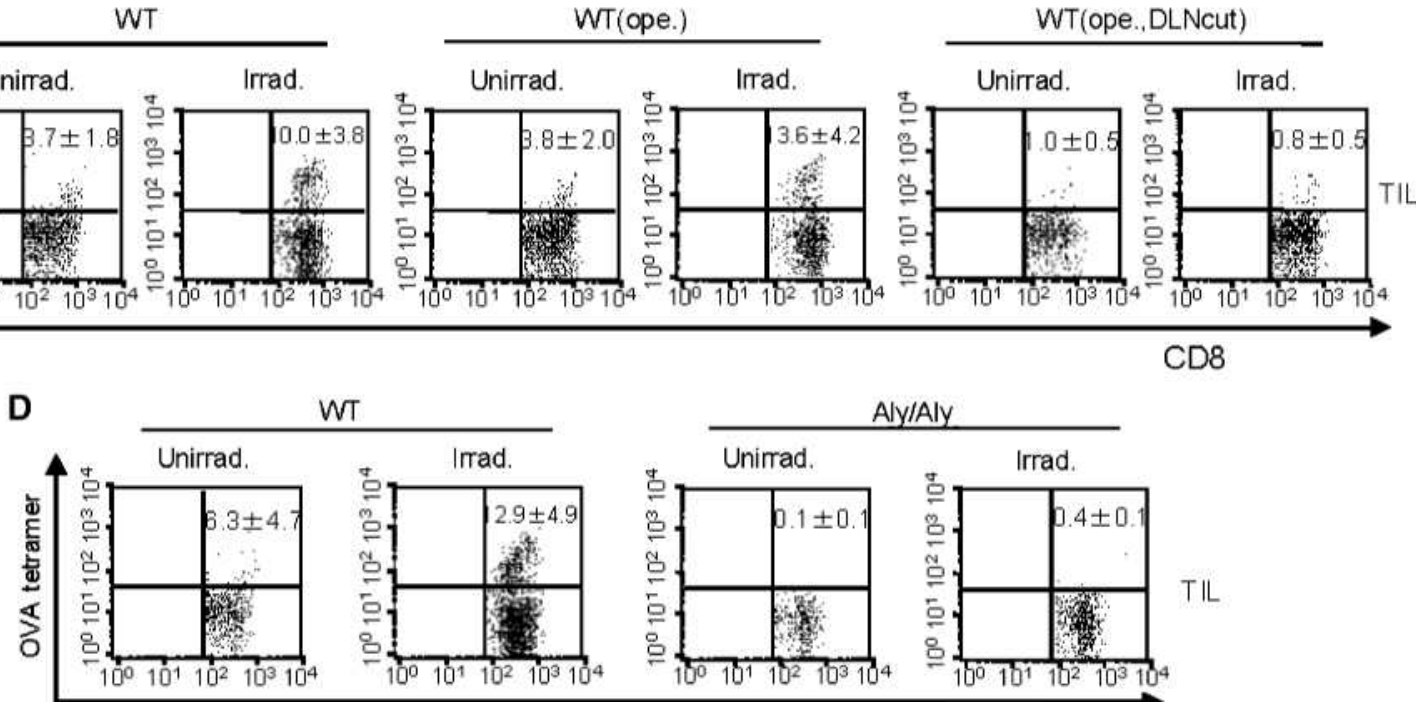

$\mathrm{CD} 8$

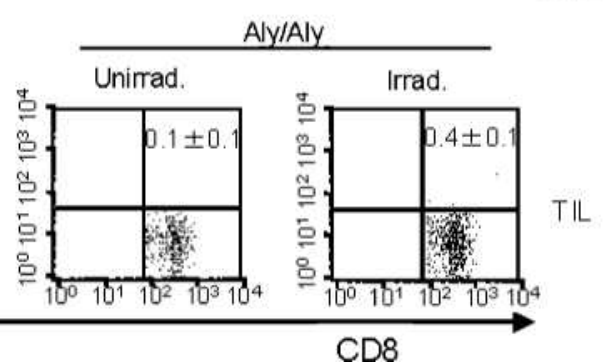

Figure 4. Tumor DLN are essential for radiation therapy. A, tumor growth in surgically DLN-ablated wild-type mice. B, tumor growth in Aly/Aly mice. $\mathrm{C}$ and D, OVA-specific CTL frequency among the CD8 ${ }^{+}$T-cell population in TIL of surgically DLN-ablated wild-type mice (C) and Aly/Aly mice (D). EG7-bearing mice were irradiated $7 \mathrm{~d}$ after tumor inoculation and TIL were analyzed $5 \mathrm{~d}$ after irradiation. Representative data of three experiments; numbers in the top right quadrant represent the mean $\pm \mathrm{SD}$ percentage of tetramer ${ }^{+} \mathrm{CD}^{+} \mathrm{T}$ cells of the total $\mathrm{CD} 8^{+} \mathrm{T}$ cells of four mice in each experimental group. ${ }^{*}, P<0.05$, two-tailed Student's $t$ test.

\section{Discussion}

Radiation therapy has been used as an important local modality for cancer treatment $(1,26)$. However, this therapy cannot always achieve complete tumor regression because of $(a)$ various mechanisms by which the tumors can escape from radiation therapy, $(b)$ the oxygen state in the tumor mass, and (c) distant metastasis (26). Here, we propose that a combination treatment of local radiation therapy and Thl cell therapy is a rational strategy for complete tumor rejection.

It has been considered that local tumor irradiation kills tumor cells by directly damaging tumor DNA (26). However, recent studies have shown that radiation therapy induces tumor-specific immune responses $(2-6,27)$. Apetoh and colleagues (3) showed that T-cell immunity was necessary for a full radiation effect by comparing tumor growth between nude mice and wild-type mice. Lugade and colleagues (2) showed that radiation induced tumor-specific CTL in DLN and tumor sites. Schaue and colleagues (4) showed that survivin-specific $\mathrm{CD}^{+} \mathrm{T}$ lymphocytes were detected in the peripheral blood of some cancer patients after presurgical chemoradiotherapy or standard radiation therapy. Importantly, Lee and colleagues (6) showed that the efficacy of local irradiation therapy was dependent on $\mathrm{CD}^{+} \mathrm{T}$ cells in a B16 model. These works provide important evidence that radiotherapy activates $\mathrm{T}$-cell immunity. However, it still remains unknown whether DLN are necessary for inducing tumor-specific CTL at the tumor site and how radiation-induced CTL eradicate tumors. In this article, we clearly showed that $(a)$ local radiation induced functional tumor-specific CTL not only in a tumor model antigen system (OVA-expressing EG7 lymphoma system or LLC-OVA system) but also in a natural tumor antigenexpressing B16 melanoma system (Figs. $1 \mathrm{~A}$ and B and Figs. 3); (b) treatment of tumor-bearing mice with anti-CD8 
mAb, which depleted CTL precursor cells, before local irradiation caused an inhibition of the protective effects of radiation therapy (Figs. 2 and 3C); and (c) tumor DLN were required for inducing tumor-specific CTL in radiation therapy in our animal model because the numbers of OVA-tetramer ${ }^{+}$CTL were greatly decreased by the ablation of DLN or in LN-deficient Aly/Aly mice (Fig. 4A-D). These results show that DLN are essential for the induction of tumor-specific tetramer ${ }^{+}$CTL, which in turn are necessary for tumor inhibition during radiotherapy. In previous articles $(8,9)$, we also showed that tumor-specific CTL were not induced in LN-deficient Aly/Aly mice treated with Th1 cells or $\mathrm{CpG}$ plus tumor antigen. Therefore, as suggested by Apetoh and colleagues (5), we propose that it is necessary to reconsider DLN resection, which is routinely applied to cancer patients with radiation tumor therapy. We need further clinical studies to resolve this question in the future.

As perforin has been shown to be a critical molecule for the lysis of tumor cells (28), we next examined whether the radiation-induced CTL destroyed tumors in a perforin- dependent manner. As shown in Supplementary Fig. S5, radiation-induced antitumor immunity was partially but not totally canceled in perforin-deficient mice. This might be because lytic molecules other than perforin such as tumor necrosis factor (TNF)- $\alpha$, granzyme B, FasL, and TNF-related apoptosis-inducing ligand were also involved in CD8 ${ }^{+} \mathrm{CTL}-$ mediated cytotoxicity during radiation-induced antitumor effect (29-31). We confirmed that EG7 expressed Fas, and TIL of the radiation-treated tumor-bearing mice expressed granzyme B (data not shown). Therefore, in addition to perforin, Fas/FasL and granzyme B might conribute to our combination radiotherapy $(30,31)$.

The refractoriness of B16 melanoma cells to radiotherapy was shown by the finding that TRP-2-tetramer ${ }^{+}$B16-specific CTL were detected only in the tumor mass of B16-bearing mice after irradiation (Fig. 3A), although OVA-tetramer ${ }^{+}$ CTL were induced in the DLN and tumor mass of EG7- and LLC-OVA-bearing mice after irradiation, respectively (Fig. 1A and B). This might have been due to some

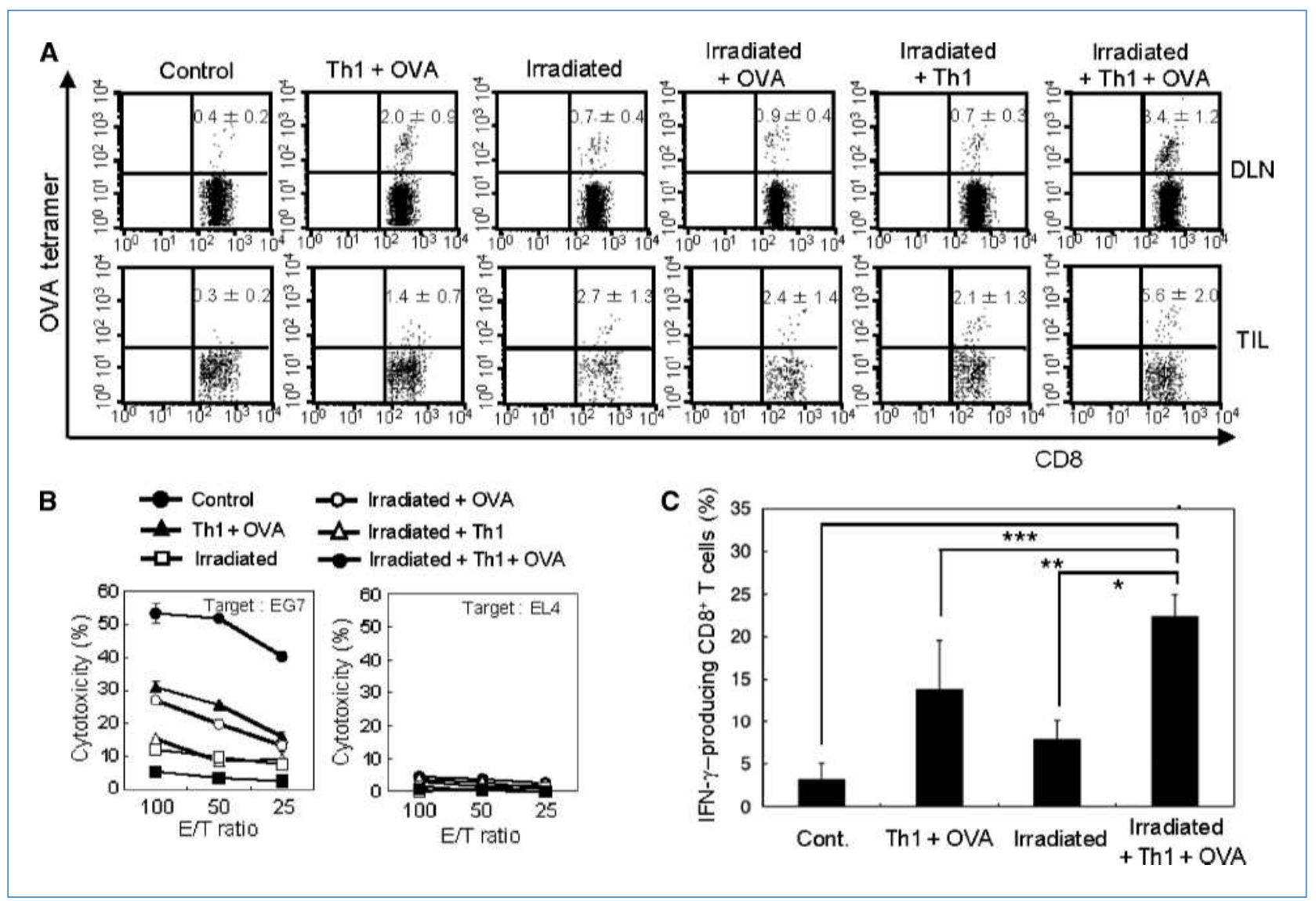

Figure 5. Synergistic effect of inducing tumor-specific CTL in tumor-bearing mice treated by local irradiation therapy combined with tumor-specific Th1 cells and tumor antigens. A, generation of tumor-specific CTL in the DLN and TIL of EG7-bearing mice treated with combination therapy of irradiation and Th1 adjuvant treatment. B, cytotoxicity of total DLN against EG7 or EL4 cells. The cytotoxicity of the total DLN cells prepared from EG7-bearing mice was measured by a $4-\mathrm{h}^{51} \mathrm{Cr}$-release assay. C, radiotherapy combined with Th1 adjuvant therapy induces IFN- $\gamma$-producing CD8 ${ }^{+}$cells. Lymphocytes were prepared from EG7-bearing mice $5 \mathrm{~d}$ after irradiation and they were stimulated with anti-CD3 mAb for $36 \mathrm{~h}$. The intracellular cytokine expression of CD8 ${ }^{+}$ T cells was examined by flow cytometry. Cont., control. The numbers in the top right quadrant represent the mean \pm SD percentage of tetramer ${ }^{+} \mathrm{CD}^{+}$ $\mathrm{T}$ cells of the total $\mathrm{CD} 8^{+} \mathrm{T}$ cells of four mice in each experimental group. Similar results were obtained from three different experiments. ${ }^{*}, P<0.05 ;{ }^{* *}$, $P<0.05 ;{ }^{* \star}, P<0.05$, two-tailed Student's $t$ test. 


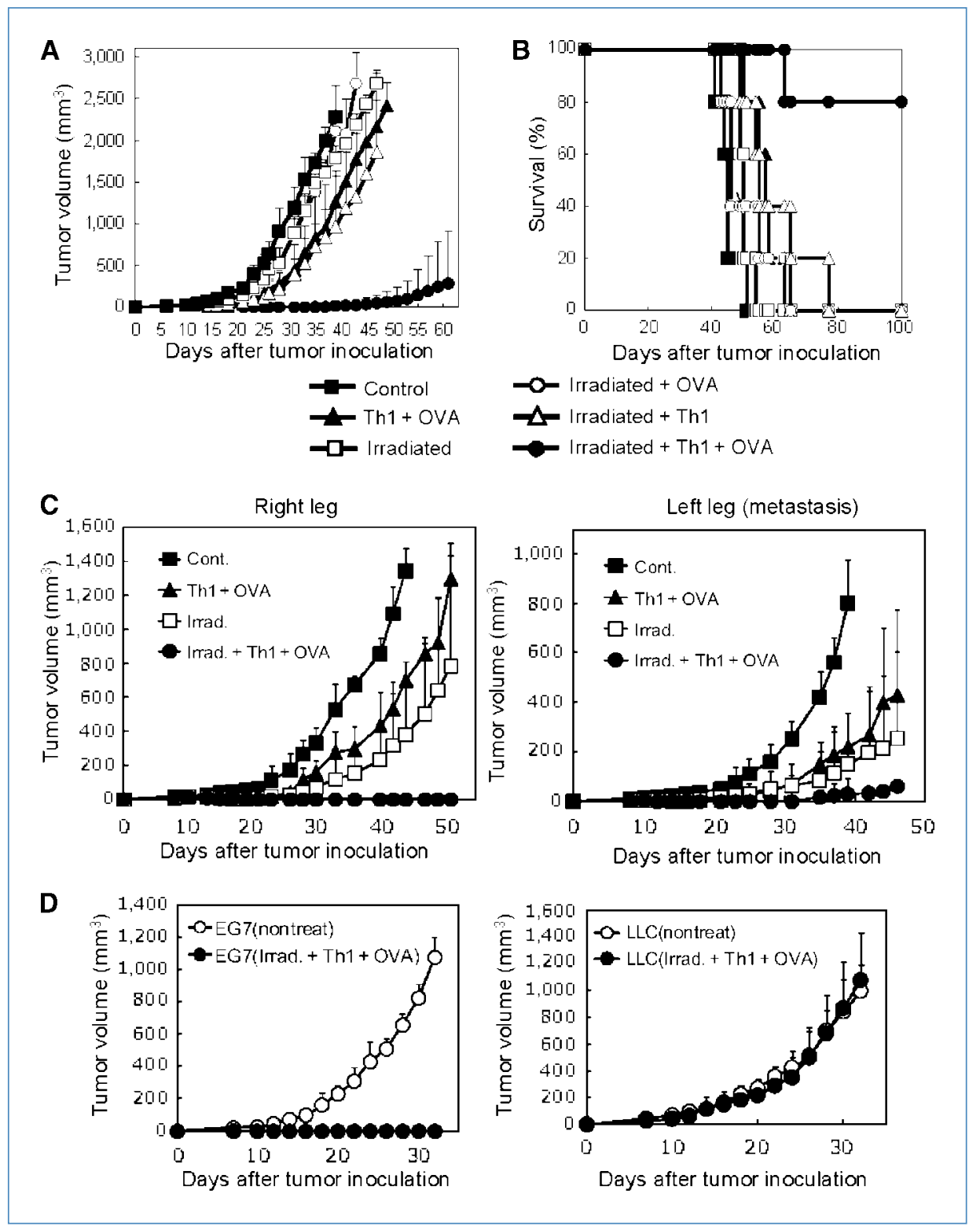

Figure 6. Eradication of EG7 tumor by local irradiation therapy combined with Th1 cell therapy. $A$ and $B$, therapeutic effect of radiotherapy combined with Th1 cell therapy. The antitumor effect was determined by measuring the tumor size $(A)$ and by assessing the survival ratio (B). C, the combination of radiotherapy and immunotherapy induces a systemic therapeutic effect. The therapeutic protocol of the metastatic model was as described in Materials and Methods. Experimental groups consisted of five mice per group. $\mathrm{D}$, the combination therapy induces immunologic memory for CTL. The mice, which were completely eradicated by combination therapy, were rechallenged with syngeneic EG7 or irrelevant LLC and tumorigenesis was monitored. Points, mean of five mice in each experimental group; bars, SD. Similar results were obtained from three different experiments. immunosuppressive mechanisms in the DLN of B16-bearing mice. Indeed, addition of Thl cytokines into the culture of DLN cells from B16-bearing mice and MMC-treated B16 cells caused the generation of TRP-2-tetramer ${ }^{+} \mathrm{CD} 8^{+} \mathrm{CTL}$ with cytotoxicity against B16 melanoma cells (Fig. 3A and B). Therefore, local radiation therapy could induce antitumor immunity even against a natural tumor antigen and if we introduce type-1 immunity into immunosuppressive states.

In an ongoing series of studies $(8,25,32,33)$, we have shown a critical role of Thl immunity in the induction of tumor-specific CTL in tumor-bearing mice. Here, we showed that local tumor irradiation is a rational strategy to augment the therapeutic effect of Thl cell therapy, inducing complete tumor regression concomitantly with efficient induction of OVA-specific CTL in the tumor DLN and tumor mass (Figs. 5 and $6 \mathrm{~A}$ and $\mathrm{B})$. In our previous article $(9,34)$, treatment of tumor-bearing mice with $\mathrm{CpG}$ or recombinant IL-12 also induced tumor-specific Th1-dominant immunity. Judging from these results, it may be possible to potentiate the therapeutic efficacy of radiation therapy by continuous administration of CpG or IL-12 instead of Th1 cells. In addition, we found that combined therapy induced a systemic antitumor immunity to prevent distal tumor masses and generated immunologic memory to reject rechallenged tumors (Fig. 6C and D), indicating that this strategy would be applicable for preventing metastasis and recurrence.

We showed that the induction of OVA-tetramer ${ }^{+} \mathrm{CD}^{+}$ $\mathrm{T}$ cells was not inhibited by anti-IFN- $\gamma$ mAb administration (Supplementary Figs. S4 and S5). These results are inconsistent with the report of Lugade and colleagues (27) who 
concluded that IFN- $\gamma$ was instrumental in creating a tumor microenvironment conducive for T-cell infiltration and tumor cell recognition. It has been accepted that IFN- $\gamma$ is a very important cytokine for triggering antitumor immunity to induce tumor-specific CTL in immunosuppressed tumor microenvironments $(32,35)$. However, it is also true that IFN- $\gamma$ is not always essential for inducing CTL because other type I IFN such as IFN- $\alpha$ and IFN- $\beta$ could compensate for the defect of IFN- $\gamma(9,36)$. Therefore, it might be possible that there were distinct requirements of IFN- $\gamma$ for inducing antitumor immunity against B16 melanoma cells or EG7 tumor in radiotherapy. It has been reported that B16 melanoma cells, which Lugade and colleagues (27) used, were refractory to immunotherapy because of their low expression of MHC class I molecules, whereas EG7 expressing high levels of MHC class I molecules were very sensitive to radiation therapy and immunotherapy (Supplementary Fig. S6). These characteristic differences between B16 melanoma cells and EG7 tumor cells might explain why these tumors showed distinct requirements of IFN- $\gamma$ for triggering radiation-induced CTL.

We also showed that the induction of tetramer ${ }^{+}$CTL and the therapeutic effect of a combination therapy with local radiation and Thl cell therapy were not inhibited by anti-IFN- $\gamma$ mAb administration (Supplementary Figs. S4 and S5). Our results are supported by Berner and colleagues (37), who showed that triggering of IFN- $\gamma$-producing $\mathrm{CD}^{+} \mathrm{T}$ cells in tumorbearing mice by injection with anti-CD40 antibody and IL-2 induced the apoptosis of $\mathrm{CD}^{+} \mathrm{T}$ cells in an IFN- $\gamma$-dependent manner, which caused the reduction of CTL memory antitumor responses. In contrast, treatment of IFN- $\gamma$ receptor-deficient tumor-bearing mice with anti-CD40 and IL-2 caused the in-

\section{References}

1. Shirato H, Shimizu S, Kitamura K, Onimaru R. Organ motion in imageguided radiotherapy: lessons from real-time tumor-tracking radiotherapy. Int J Clin Oncol 2007;12:8-16.

2. Lugade A, Moran J, Gerber S, Rose R, Frelinger J, Lord E. Local radiation therapy of B16 melanoma tumors increases the generation of tumor antigen-specific effector cells that traffic to the tumor. J Immunol 2005;174:7516-23.

3. Apetoh L, Ghiringhelli F, Tesniere A, et al. Toll-like receptor 4-dependent contribution of the immune system to anticancer chemotherapy and radiotherapy. Nat Med 2007;13:1050-9.

4. Schaue D, Comin-Anduix B, Ribas A, et al. T-cell responses to survivin in cancer patients undergoing radiation therapy. Clin Cancer Res 2008;14:4883-90.

5. Apetoh L, Ghiringhelli F, Tesniere A, et al. The interaction between HMGB1 and TLR4 dictates the outcome of anticancer chemotherapy and radiotherapy. Immunol Rev 2007;220:47-59.

6. Lee $\mathrm{Y}$, Auh $\mathrm{S}$, Wang $\mathrm{Y}$, et al. Therapeutic effects of ablative radiation on local tumor require $\mathrm{CD}^{+} \mathrm{T}$ cells: changing strategies for cancer treatment. Blood 2009;114:589-95.

7. Chamoto K, Takeshima T, Wakita D, et al. Combination immunotherapy with radiation and CpG-based tumor vaccination for the eradication of radio- and immuno-resistant lung carcinoma cells. Cancer Sci 2009;100:934-9.

8. Chamoto $\mathrm{K}$, Wakita $\mathrm{D}, \mathrm{Narita} \mathrm{Y}$, et al. An essential role of antigenpresenting cell/T-helper type 1 cell-cell interactions in draining lymph node during complete eradication of class II-negative tumor tissue by T-helper type 1 cell therapy. Cancer Res 2006;66:1809-17.

9. Wakita $D$, Chamoto $K$, Zhang $Y$, et al. An indispensable role of type-1 crease of $\mathrm{CD}^{+} \mathrm{T}$ cells and induction of CTL memory antitumor responses in the absence of IFN- $\gamma(37)$. Thus, unknown Th1-derived cytokines, in addition to IFN- $\gamma$, might be also involved in CTL-mediated antitumor immunity during our combined radiotherapy.

Recently, it has also been reported that chemotherapy against tumors was beneficial for inducing CTL $(38,39)$. Therefore, we should also consider the potential efficacy of combination therapy consisting of rational immunotherapy and primary cancer treatment modalities including radiation therapy, chemotherapy, and surgical therapy to prevent the recurrence of cancer.

\section{Disclosure of Potential Conflicts of Interest}

No potential conflicts of interest were disclosed.

\section{Acknowledgments}

We thank Takuko Sawada (Shionogi Pharmaceutical Institute Co., Osaka, Japan) for her kind donation of IL-2.

\section{Grant Support}

Grant-in-Aid for the National Project "Knowledge Cluster Initiative" (2nd stage, "Sapporo Biocluster Bio-S") from the Ministry of Education Culture, Sports, Science, and Technology (MEXT), a Grant-in-Aid for Young Scientists (B) from MEXT, and The Matching Program for Innovations in Future Drug Discovery and Medical Care from MEXT of the Japanese Government.

The costs of publication of this article were defrayed in part by the payment of page charges. This article must therefore be hereby marked advertisement in accordance with 18 U.S.C. Section 1734 solely to indicate this fact.

Received 08/11/2009; revised 01/18/2010; accepted 01/19/2010; published OnlineFirst 03/09/2010.
IFNs for inducing CTL-mediated complete eradication of established tumor tissue by CpG-liposome co-encapsulated with model tumor antigen. Int Immunol 2006;18:425-34.

10. Song K, Chang Y, Prud'homme G. Regulation of T-helper-1 versus T-helper-2 activity and enhancement of tumor immunity by combined DNA-based vaccination and nonviral cytokine gene transfer. Gene Ther 2000;7:481-92.

11. Santin A, Hermonat $P$, Ravaggi $A$, et al. Interleukin-10 increases Th1 cytokine production and cytotoxic potential in human papillomavirusspecific CD8(+) cytotoxic T lymphocytes. J Virol 2000;74:4729-37.

12. Casares N, Lasarte J, de Cerio A, et al. Immunization with a tumorassociated CTL epitope plus a tumor-related or unrelated Th1 helper peptide elicits protective CTL immunity. Eur J Immunol 2001;31: 1780-9.

13. Chang J, Choi M, Cheong H, Kim K. Development of Th1-mediated $\mathrm{CD}^{+}$effector $\mathrm{T}$ cells by vaccination with epitope peptides encapsulated in pH-sensitive liposomes. Vaccine 2001;19:3608-14.

14. Mashino K, Sadanaga N, Tanaka F, Ohta M, Yamaguchi H, Mori M. Effective strategy of dendritic cell-based immunotherapy for advanced tumor-bearing hosts: the critical role of Th1-dominant immunity. Mol Cancer Ther 2002;1:785-94.

15. Emtage P, Clarke D, Gonzalo-Daganzo R, Junghans R, Gonzalo-Dagonzo R. Generating potent Th1/Tc1 T cell adoptive immunotherapy doses using human IL-12: harnessing the immunomodulatory potential of IL-12 without the in vivo-associated toxicity. J Immunother 2003;26: 97-106.

16. Nakamori $M$, Iwahashi $M$, Nakamura $M$, Ueda $K$, Zhang $X$, Yamaue $H$. Intensification of antitumor effect by $T$ helper 1-dominant adoptive 
immunogene therapy for advanced orthotopic colon cancer. Clin Cancer Res 2003;9:2357-65.

17. Tsuji $\mathrm{T}$, Chamoto $\mathrm{K}$, Funamoto $\mathrm{H}$, et al. An efficient method to prepare $T$ cell receptor gene-transduced cytotoxic T lymphocytes type 1 applicable to tumor gene cell-therapy. Cancer Sci 2003;94:389-93.

18. Harada M, Matsueda S, Yao A, Noguchi M, Itoh K. Vaccination of cytotoxic $T$ lymphocyte-directed peptides elicited and spread humoral and Th1-type immune responses to prostate-specific antigen protein in a prostate cancer patient. J Immunother 2005;28: 368-75.

19. Chattopadhyay S, Chakraborty N. Continuous presence of Th1 conditions is necessary for longer lasting tumor-specific CTL activity in stimulation cultures with PBL. Hum Immunol 2005;66:884-91.

20. Miyawaki S, Nakamura $Y$, Suzuka $H$, et al. A new mutation, aly, that induces a generalized lack of lymph nodes accompanied by immunodeficiency in mice. Eur J Immunol 1994;24:429-34.

21. Walsh $C$, Matloubian $M$, Liu $C$, et al. Immune function in mice lacking the perforin gene. Proc Natl Acad Sci U S A 1994;91:10854-8.

22. Matsuzaki J, Tsuji T, Chamoto $K$, Takeshima T, Sendo F, Nishimura $T$. Successful elimination of memory-type $C D 8^{+} T$ cell subsets by the administration of anti-Gr-1 monoclonal antibody in vivo. Cell Immunol 2003;224:98-105.

23. Nishimura $T$, Burakoff $S$, Herrmann $S$. Protein kinase $C$ required for cytotoxic T lymphocyte triggering. J Immunol 1987;139:2888-91.

24. Yokouchi $H$, Chamoto $K$, Wakita $D$, et al. Tetramer-blocking assay for defining antigen-specific cytotoxic $\mathrm{T}$ lymphocytes using peptideMHC tetramer. Cancer Sci 2006;97:148-54.

25. Zhang $Y$, Wakita $D$, Chamoto $K$, et al. Th1 cell adjuvant therapy combined with tumor vaccination: a novel strategy for promoting CTL responses while avoiding the accumulation of Tregs. Int Immunol 2007;19:151-61.

26. Bernier J, Hall E, Giaccia A. Radiation oncology: a century of achievements. Nat Rev Cancer 2004;4:737-47.

27. Lugade A, Sorensen E, Gerber S, Moran J, Frelinger J, Lord E. Radiation-induced IFN- $\gamma$ production within the tumor microenvironment influences antitumor immunity. J Immunol 2008;180:3132-9.
28. Kägi D, Ledermann B, Bürki K, et al. Cytotoxicity mediated by $T$ cells and natural killer cells is greatly impaired in perforin-deficient mice. Nature 1994;369:31-7.

29. Barry M, Bleackley R. Cytotoxic T lymphocytes: all roads lead to death. Nat Rev Immunol 2002;2:401-9.

30. Shanker A, Brooks A, Jacobsen $K$, et al. Antigen presented by tumors in vivo determines the nature of $\mathrm{CD}^{+} \mathrm{T}$-cell cytotoxicity. Cancer Res 2009;69:6615-23.

31. Kim H, Lee Y, Kim D. Doxorubicin exerts cytotoxic effects through cell cycle arrest and Fas-mediated cell death. Pharmacology 2009; 84:300-9.

32. Nishimura $T$, Iwakabe $K$, Sekimoto $M$, et al. Distinct role of antigenspecific Thelper type 1 (Th1) and Th2 cells in tumor eradication in vivo. J Exp Med 1999;190:617-27.

33. Ikeda $\mathrm{H}$, Chamoto $\mathrm{K}$, Tsuji $\mathrm{T}$, et al. The critical role of type-1 innate and acquired immunity in tumor immunotherapy. Cancer Sci 2004 95:697-703.

34. Nakui M, Ohta A, Sekimoto $M$, et al. Potentiation of antitumor effect of NKT cell ligand, a-galactosylceramide by combination with IL-12 on lung metastasis of malignant melanoma cells. Clin Exp Metastasis 2000;18:147-53.

35. Trinchieri G, Pflanz S, Kastelein R. The IL-12 family of heterodimeric cytokines: new players in the regulation of $\mathrm{T}$ cell responses. Immunity 2003;19:641-4.

36. Janssen $\mathrm{E}$, Tabeta $\mathrm{K}$, Barnes $\mathrm{M}$, et al. Efficient $\mathrm{T}$ cell activation via a Toll-interleukin 1 receptor-independent pathway. Immunity 2006;24: 787-99.

37. Berner V, Liu $\mathrm{H}$, Zhou $\mathrm{Q}$, et al. IFN- $\gamma$ mediates $C D 4^{+} \mathrm{T}$-cell loss and impairs secondary antitumor responses after successful initial immunotherapy. Nat Med 2007;13:354-60.

38. Lake R, Robinson B. Immunotherapy and chemotherapy-a practical partnership. Nat Rev Cancer 2005;5:397-405.

39. Correale P, Del Vecchio M, La Placa M, et al. Chemotherapeutic drugs may be used to enhance the killing efficacy of human tumor antigen peptide-specific CTLs. J Immunother 2008;31:132-47. 